\title{
Stopping Power Calculation of Protons using Bloch Correction and Semi Empirical Relation
}

\author{
Dr. Rashid Owaid Kadhim¹, Ahlam Habeeb Hussien² \\ University of Kufa /College of Education for Girls
}

\begin{abstract}
One of the needs of various accelerators to stimulate recent developments in calculating the energy loss of charged particles in matter is heavy ion ionization energy loss. Bloch incorporates Bethe and Bohr theory in a very transparent way. Thus, Bloch found the bridging formulation between the classical Bohr impact -parameter approach and the quantized Bethe momentum transfer approach to energy loss. In this paper, the behavior of Bloch correction of protons in $\left(\mathrm{C}_{3} \mathrm{H}_{6}, \mathrm{C}_{22} \mathrm{H}_{10} \mathrm{~N}_{2} \mathrm{O}_{5}\right)$ and for the energy range (0.1- 1000) MeV has been studied. We have described an implementation of Bloch correction to stopping power ( $d E / d x)$ in order to take into account the deviations from the Bethe theory at non - relativistic and relativistic velocities as well as the effects at ultra relativistic velocities. Bloch correction is expressed in terms of the transport cross section for electron - ion scattering and dependence on the scattering phase shifts. Were calculated stopping power equation by quasi-experimental results showed using both methods showed good agreement with the results SRIM 2012.
\end{abstract}

Keywords: effective of Bloch, stopping power

\section{Introduction}

The electronic energy loss has been studied for many years because of its direct application in problems concerning material damage, ion beam analysis and plasma physics. The theoretical treatment of the energy loss in atomic collisions has been greatly improved over the last decades[1]. Stopping power, i.e. energy loss of energetic particles per unit length in matter, has been studied experimentally and theoretically since the beginning of the 20th century because of its wide application area, such as ion implantation, fundamental particle physics, nuclear physics, radiation damage, radiology, and structure analysis of solid target by Rutherford backscattering spectroscopy [2]. The slowing down of energetic ions in matter is dominated by momentum exchanging collision with electrons. The theory of this venerable subject was formulated early on by Bohr [3] and Bethe[4]. The first classical calculation of the energy loss of energetic particles was made by Bohr[3], while the first quantum mechanical treatment was done by Bethe[4]. This latter theory of stopping power is particularly accurate when the projectile's velocity is sufficiently high. It was modified by Bloch [5], and it was shown that at relativistic velocities a Mott correction for spin changing collisions was required[6]. Lindhard and Sorensen performed exact quantum mechanical calculations on the basis of the Dirac equation to produce values for the average energy loss and straggling which are stated to be accurate for any value of projectile charge[7].

\section{Stopping Power}

The mean ionization energy loss of charged particles heavier than electrons is given by the Bethe expression[8]

$$
-\frac{1}{\rho} \frac{d E}{d x}=4 \pi N_{a} r_{\varepsilon}^{2} m c^{2} \frac{z_{1}^{2} z_{2}}{A_{1} A_{2}} \frac{1}{\beta^{2}} L(\beta) \text { (1) }
$$

Where

$L(\beta)$ is the ionization logarithm and presented in the following form

$$
\begin{gathered}
L(\beta)=L_{0}(\beta)+\sum_{i} \Delta L_{i} \\
L_{0}=\ln \left(\frac{2 m c^{2} \beta^{2} \gamma^{2}}{I}\right)-\beta^{2}-\frac{\delta}{2}
\end{gathered}
$$

$\gamma=\left(1-\beta^{2}\right)^{-1 / 2}$ is the Lorentz factor

$r_{e}=\frac{\varepsilon^{2}}{4 \pi E_{0} m c^{2}}$ is the classical electron radius, $\beta=\frac{v}{c}$ is the velocity of the particle relative to light velocity.

$Z_{1}$ and $A_{1}$ are the atomic and mass of the projectile, $Z_{2}$ and $A_{2}$ are the atomic and mass of the target material, $I$ and $\delta$ are the mean excitation energy and density correction, respectively, $N_{a}$ is Avogadro's number.

When neglecting all the corrections $\Delta \mathrm{L}$ and dealing only with the $L_{0}(\beta)$, eq.(1) is referred to as the Bethe equation. The corrections $\Delta \mathrm{L}$ are to take into account the deviations from the Bethe theory for ions at both low and high energies[8]. This is the form derived originally from the quantum perturbation theory, and the first two terms are typically called the Bethe result. The third terms is the density effect. We will refer to as the Bethe result inclusive of the density effect[9]. At high energies, the electronic stopping is determined by Bethe's or Bohr's treatment [10]. The decisive point is whether or not the problem can be treated by classical theory. This is possible for large values of Bohr's parameter [11].

$$
K=\frac{2 z_{1} v_{0}}{v}=2 \eta
$$

In the quantum mechanical limit $K \ll 1$ Bethe's formula based on a first order perturbation treatment gives the high energy expression in the non relativistic case valid for light ions[10]

$$
L_{\text {Bethe }}=\ln \frac{2 m v^{2}}{1}
$$

Once notes that the condition for quantum can not be expected to hold for slow projectiles or projectiles of high atomic number. In this case the logarithm in eq.(4) is replaced by Bohr's expression[10]

$$
L_{\text {Boh } r}=\ln \frac{c m v^{3}}{z_{1} \epsilon^{2}}
$$




\section{International Journal of Science and Research (IJSR) \\ ISSN (Online): 2319-7064}

Index Copernicus Value (2013): 6.14 | Impact Factor (2014): 5.611

$\mathrm{C}=2 e^{-\gamma^{-}}=1.1229 ; \gamma^{-}=0.5772$ is (Euler's constant).

The major difference is that enters into the argument of the logarithm, and therefore, influences the position of the stopping maximum. is a characteristic frequency of each electron level of the target atom, since the derivation is performed for a harmonic oscillator[10].

\section{Bloch Correction $(\Delta \mathrm{L})$}

Bloch indicated the exact scattering amplitude (but non relativistic) should be adopted for collisions with small impact parameter, and derived a correction which is called $\left(\mathrm{Z}_{1}^{4}\right)$ correction term[12]

$$
\Delta L_{\text {Bloch }}=Z_{1} L_{2}=\Psi(1)-\operatorname{Re} \Psi\left(1+i \frac{z_{1} v_{0}}{v}\right)
$$

Here $\Psi(x)=d \ln \Gamma(x) / d x$ is the digamma function, $v_{0}$ is the Bohr velocity and with $y=\frac{z_{1} v_{0}}{v}$, eq.(6) becomes

$$
\Delta L_{\text {Bloch }}=Z_{1} L_{2}=\Psi(1)-R_{\theta} \Psi(1+i y)
$$

To bridge the gap between the Bethe's quantum mechanical and Bohr's classical formula. This term is mainly originated in the close collision with small scattering angle which is important for low velocity ions[12]. Eq.(6) may be as a chain

$$
\begin{gathered}
\Delta L_{\text {Bloch }}=Z_{1} L_{2}=-y^{2} \sum_{n=1}^{\infty}\left[n\left(n^{2}+y^{2}\right)\right]^{-1} \\
\text { for } \mathrm{y}>1
\end{gathered}
$$

Where $n=1,2,3, \ldots \ldots$ and $\xi$ is the Rieman function From a practical viewpoint of calculating accurate stopping powers, Bichsel has proposed simple parameterization of the Bloch correction which accurately fits a wide range of high velocity stopping data[13]

$\Delta L_{\text {Bloch }}=Z_{1} L_{2}=-y^{2}\left[1.202-y^{2}\left(1.042-0.855 y^{2}+\right.\right.$

For low velocity, the value of $\left(\Delta L_{\text {Bioch }}\right)$ becomes

$$
\Delta L_{\text {Bloch }} \rightarrow 0.58-\ln (y)
$$

And thus the Bloch correction provides the transition to the classical stopping formula of Bohr.

For high velocities, i. e. $\mathrm{y} \rightarrow 0$, the value of $\left(\Delta L_{\text {Bloch }}\right)$ becomes

$$
\Delta L_{\text {Bloch }}=-1.202 y^{2}
$$

This term is usually quite small. According to Bloch, the stopping cross section of an atom is determined by the following expression for the stopping number[5]

$L_{\text {Bloch }}=L_{\text {Behe }}+\Delta L_{\text {Bloch }}$

$L_{\text {Bloch }}=\ln \frac{2 m v^{2}}{l}+\Psi(1)-R_{e} \Psi\left(1+i \frac{z_{1} v_{0}}{v}\right)$

therefore, 9 the stopping power that includes the Bloch correction is written as:

$$
-\frac{1}{\rho} \frac{d E}{d x}=4 \pi N_{a} r_{\varepsilon}^{2} m c^{2} \frac{z_{1}^{2} z_{2}}{A_{1} A_{2}} \frac{1}{\beta^{2}} L_{\text {Bloch }}
$$

When Bloch correction ( $\Delta L_{\text {Bloch }}$ ) is added to ( $L_{\text {Bethe }}$ ), one arrives at the Bohr stopping formula at the low - velocity end. $\Delta L_{\text {Bloch }}$ reduces to the Bohr logarithm eq.(5) at low projectile velocity and at high projectile velocity, $\Delta L_{\text {Bloch }}$ goes to zero[3].

\section{SRIM 2012 Program}

It is a number of experiments that are related to the measurement of the stopping power and ionic ranges in the elements. It was designed by Ziegler and his group[14] .And because of the high range of power usage which is used in the low, medium, and high power areas, it has spread very fast. Lots of improvements have been added since its first presentation in 1985, and the vehicles stopping power have been improved as well. 2800 new stopping power have been added to the date base of the SRIM program which made it more than 28000 stopping power in the SRIM data base. The new stopping power of the heavy ions emphasized the important enhancements for the precision of the stopping power in the SRIM 2012 program.

\section{Semi Empirical Relation for Stopping Power Calculation}

Many of the empirical and theoretical studies were performed in order to form standard energy and range relation to account for stopping power. The subject had been reviewed by some researchers like Taylor, Bethe, Askin, Allision, Warshaw, Uehling, Barkas and Berger in the last decades (50-60). And most of the empirical data had been collected by Whaling Bichsel in the form of tables

A. K. Chaubey and H. V. Gupta arrived at the following empirical relation for the stopping power of protons[15]:

$$
-\frac{d E}{p d x}=\frac{a}{A_{2}} E^{-b} Z_{2}^{c l o g E+d}
$$

The appropriate values of the constants a, b, c, $d$ are $a=915$, $\mathrm{b}=0.85, \mathrm{c}=0.145, \mathrm{~d}=0.635$, while $\rho$ is the density, $\mathrm{A}_{2}$ is the atomic weight and $\mathrm{Z}_{2}$ is the atomic number for the target and $0.34 \mathrm{is}^{2}$ thle kinetic energy of the particle by the units MeV/amu.

The eq(14)is found to be valid in the energy range(0.712) MeV/amu, the stopping power is in $\mathrm{MeV} \mathrm{cm} / \mathrm{gm}$. The constants c, d are found to be in dependant of particle type and were obtained by fitting Northcliffe and Schilling[16] (which was called NS later), it accounted the stopping power by the least squares method while a, b were extracted from the empirical data by Whaling[17] and Anderson et al.[18] as well as NS data in the low energies.

The stopping power for the ions heavier than protons can be found by the expressions given by Blann and pierce[19]:

$$
\left(-\frac{d E}{\rho d x}\right)_{H}=\frac{z_{a f f}^{2}}{\delta_{\bar{p}}^{2}}\left(-\frac{d E}{\rho d x}\right)_{p}
$$

Where $Z_{\text {eff }}^{2}=\delta^{2} Z^{2}$, the energy is above $0.7 \mathrm{MeV}$ here, so for protons we have taken $\delta_{p}=1$ by compensating(14) in (15) we get:

$$
\left(-\frac{d E}{\rho d x}\right)_{H}=\gamma^{2} Z^{2} a E^{-b} Z_{2}^{c \log E+D}
$$

In eq.(16) the fractional effective charge $\gamma$ of the ion of energy $\mathrm{E}(\mathrm{MeV} / \mathrm{amu})$ can be guessed from the empirical formula of Grant and Booth[20]: $\delta^{2}=f\left(E Z^{-4 / 3}\right)$

Where

$$
f(x)=1-\exp \left(-24.73 x+247.6 x^{2}-1131 x^{3}\right)
$$




\section{International Journal of Science and Research (IJSR) \\ ISSN (Online): 2319-7064}

Index Copernicus Value (2013): 6.14 | Impact Factor (2014): 5.611

For the calculation of the stopping power of alpha particles from the equation (16), we assume that the energy is bigger than $4 \mathrm{MeV}$ and the fractional effective charge of the alpha particles $\delta^{2}=1$. and when the energy is less than $4 \mathrm{MeV}$ or 0.7 to $1 \mathrm{MeV} / \mathrm{amu}$, by fitting NS data with alpha particles by the least squares method and keeping $\mathrm{c}$ and $\mathrm{d}$ as constants we get $a=3574, b=0.84$ so the energy between 0.7 to 1 MeV/amu for Alpha particles and the equation (14) should be modified into:

$$
\left(-\frac{d E}{\rho d x}\right)=\frac{3574}{A_{2}} E^{-0.84} Z_{2}^{0.145 \log E+0.635}
$$

We can get the stopping power in the compound targets by the summation theory:

$$
\begin{aligned}
& \left(-\frac{d E}{\rho d x}\right)_{\text {compound }} \\
& =\frac{1}{M_{2}} \sum_{i} N_{i} A_{i}\left(-\frac{d E}{\rho d x}\right)_{i}
\end{aligned}
$$

Where $\mathrm{M}_{2}$ is the molecular weight of the compound medium containing $N_{i}$ atoms of atomic weight $A_{i}$.

In addition to, Anderson and Ziegler[21] summarized the empirical data of proton stopping power to many elements of wide range energy. And to get the values for all the elements in a continuous range of protons energies, the researcher had fitted the curves with the available empirical data to generate Coefficient $\mathrm{e}$ to be used in the semi empirical stopping power as a function to the proton energy $\mathrm{E}(\mathrm{keV})$ and the atomic number $\mathrm{Z}_{2}$ to the target. It was assumed that $S_{e}$ to be fit with $\mathrm{E}^{0.45}$ to the energy $\mathrm{E}<25 \mathrm{keV}$, except $Z_{2} \leq 6$ which is fit with $E^{0.25}$ Zielger el al. used the following relation for $(25 \mathrm{keV} \leq \mathrm{E} \leq 10 \mathrm{MeV})$ :

$$
\begin{array}{r}
\frac{1}{s_{z}}=\frac{1}{s_{\text {LOW }}}+\frac{1}{s_{\text {HIGH }}} \\
S_{\text {LOW }}=A_{1} E^{A_{2}}+A_{3} E^{A_{4}} \\
S_{\text {HIGH }}=\frac{A_{5} \ln \left(\frac{A_{6}}{E}+A_{7} E\right)}{E^{A_{8}}}
\end{array}
$$

And for the energy rang $10 \mathrm{MeV} \leq \mathrm{E} \leq 2 \mathrm{GeV}$ repuires to use the following relation:

$$
S_{e}=A_{9}+A_{10}\left(\frac{\ln E}{E}\right)+A_{11}\left(\frac{\ln E}{E}\right)^{2}+A_{12}\left(\frac{E}{\ln E}\right)
$$

And the Coefficiente $A_{I}$ for every $Z_{2}$ is available in the form of TRIM tables[22].

In this research, The following semi experimental relationships were concluded in order to calculate protons stopping power in the compounds $\mathrm{C}_{3} \mathrm{H}_{6}$ and $\mathrm{C}_{22} \mathrm{H}_{10} \mathrm{~N}_{2} \mathrm{O}_{5}$ :-

Table 1

\begin{tabular}{|c|c|c|}
\hline Compound & $\begin{array}{c}\text { Semi Experimental } \\
\text { Relation }\end{array}$ & Constants \\
\hline $\mathrm{C}_{3} \mathrm{H}_{6}$ & $S_{e}=a e^{b E}+c e^{d E}$ & $\mathrm{a}=0.7729$ \\
& & $\mathrm{~b}=-3.321$ \\
& & $\mathrm{c}=0.2381$ \\
& & $\mathrm{~d}=-0.2002$ \\
\hline $\mathrm{C}_{22} \mathrm{H}_{10} \mathrm{~N}_{2} \mathrm{O}_{5}$ & $S_{e}=a e^{b E}+c e^{d E}$ & $\mathrm{a}=0.8226$ \\
& & $\mathrm{~b}=-3.342$ \\
& & $\mathrm{c}=0.2554$ \\
& & $\mathrm{~d}=-0.2196$ \\
\hline
\end{tabular}

\section{The Correlation Coefficient}

The Program Curve Expert 1.3 was used, the program through which the Correlation Coefficient is calculated and which in concluded to be $\left(x_{i}, y_{i}\right) i=1, \ldots . n$, a sample of the organized pairs that provides the values of two random variables on (n) of the elements. This factor treats the link between the variables $x$ and $y$ and is referred to as $r=r(x, y)$ and which calculated as follows[23]:

1) When $r>0$ then there is a linear link.

2) When $r<0$ then there is a reverse link.

3) When $r=1$ then there is a full extrusive linear link.

4) When $r=-1$ then there is a full reverse linear link.

5) When $r=0$ then there is no linear link.

6) Whenever the absolute value goes farther than 1 , the linear link goes weaker

\section{Result and Discussion}

The figure(1-a) shows the results of stopping power as a function of energy for protons in $\mathrm{C}_{3} \mathrm{H}_{6}$ and The figure(1-b) shows the results of stopping power as a function of energy for protons in $\mathrm{C}_{22} \mathrm{H}_{10} \mathrm{~N}_{2} \mathrm{O}_{5}$

Fig (1) shows a great convergence between the variables of Bloch equation of the experimental variables of the SRIM 2012 program except the first variable the kapton compound $\mathrm{C}_{22} \mathrm{H}_{10} \mathrm{~N}_{2} \mathrm{O}_{5}$ of the starting point of the energy range $0.1 \mathrm{MeV}$ because of the reduction in the values of the stopping numbers of Bethe according to the equation (13). That affected the negative Bloch correction values which led to the stopping power values of the Bloch equation. And the greatest stopping power at the value $0.18 \mathrm{MeV}$ and at the values higher than $0.18 \mathrm{MeV}$, the stopping power are higher than the results of the SRIM 2012 according to Bloch which indicates that irritation and ionization. Then, we notice a very close convergence between the values of the Bloch formula and the experimental values SRIM 2012 until the end of the used range $1000 \mathrm{MeV}$.

Figure (2) shows the results of stopping power as a function of energy for protons in $\mathrm{C}_{3} \mathrm{H}_{6}$ and $\mathrm{C}_{22} \mathrm{H}_{10} \mathrm{~N}_{2} \mathrm{O}_{5}$, when performing a comparison between of the tow mediums for the same projectile we would find the stopping power is that of the multe-broblin compound $\mathrm{C}_{3} \mathrm{H}_{6}$ highest from the Kapton compound $\mathrm{C}_{22} \mathrm{H}_{10} \mathrm{~N}_{2} \mathrm{O}_{5}$, and that it this is because of two factors:

\section{1) Average Ionization Potential $\langle I\rangle$}

Considering the variety of the average ionization potential gose to the atomic number $\mathrm{Z}_{\mathrm{i}}$ for each element of the compound along its percentage $n_{i}$ in the compound itself. The multi-broblin compound $\mathrm{C}_{3} \mathrm{H}_{6}$ has a certain ionization potential $(\mathrm{I}=56.5429 \quad \mathrm{eV})$ and the kapton compound $\mathrm{C}_{22} \mathrm{H}_{10} \mathrm{~N}_{2} \mathrm{O}_{5}$ has a certain ionization potential as well $(\mathrm{I}=80.368 \mathrm{eV})$, and since it is the denominator, it is inversely proportional with the stopping power.

\section{2) The Fraction $\frac{z_{z}}{A_{z}}$}

The fraction includes the atomic and the mass numbers of the element with its percentage in the compound itself $\left(Z_{i}, A_{i}\right.$, 


\section{International Journal of Science and Research (IJSR) \\ ISSN (Online): 2319-7064}

Index Copernicus Value (2013): 6.14 | Impact Factor (2014): 5.611

$\mathrm{n}_{\mathrm{i}}$ ) It is for the multi-broblin compound $\mathrm{C}_{3} \mathrm{H}_{6}(0.5714)$ and the Kapton compound $\mathrm{C}_{22} \mathrm{H}_{10} \mathrm{~N}_{2} \mathrm{O}_{5}(0.5131)$. And, since it is proportional with the stopping power, the stopping power of the multe-broblin $\mathrm{C}_{3} \mathrm{H}_{6}$ is greater than that of the Kapton compound $\mathrm{C}_{22} \mathrm{H}_{10} \mathrm{~N}_{2} \mathrm{O}_{5}$.

Figure (3) shows the results of Bloch correction as a function of energy for protons in different targets $\mathrm{C}_{3} \mathrm{H}_{6}\left(\mathrm{Z}_{2}=24\right)$ and $\mathrm{C}_{22} \mathrm{H}_{10} \mathrm{~N}_{2} \mathrm{O}_{5}\left(Z_{2=196)}\right.$ which are calculated from eq.(10) at high velocity. In this figure, the Bloch correction of protons is the same in two target and no variation with $Z_{2}$ because it is independent on the target and this is mathematically clear as shown in eq.(10). From the figure, the Bloch correction decreases with increasing the energy and becomes quite small and approaches to zero at very high velocity.

Figure (4) shows the percent contribution of Bloch correction to stopping number as a function of energy for protons in different targets $C_{3} H_{6}\left(Z_{2}=24\right)$ and $\mathrm{C}_{10} \mathrm{H}_{8} \mathrm{O}_{4}\left(\mathrm{Z}_{2}=100\right)$ which are calculated from eq.(10). From the figure, the percent of Bloch correction decreases with increasing the energy and becomes small at high velocity, therefore there is a divergence in values at low velocities and an convergence in values of percent contribution of Bloch correction at high velocities. The percent Bloch correction in $\mathrm{C}_{3} \mathrm{H}_{6}$ target is larger than that of $\mathrm{C}_{10} \mathrm{H}_{8} \mathrm{O}_{4}$ target because the targets are different and for each one a specific atomic number and there are a number of corrections to the stopping number for each target.

Figure (5) the Correlation Coefficient (r) and the error margin(s) were calculated between the semi experimental formula that has been concluded and which is represented in table (1) along with the results of SRIM 2012 From the preceding results, we conclude that there is only one good match according to the Correlation Coefficient.

Figure (6) the Correlation Coefficient (r) and the error margin(s) between the results of Bloch formula were also calculated and are represented by the formula (13) with the results SRIM 2012 From the preceding results, we conclude that there is only one good match according to the Correlation Coefficient.

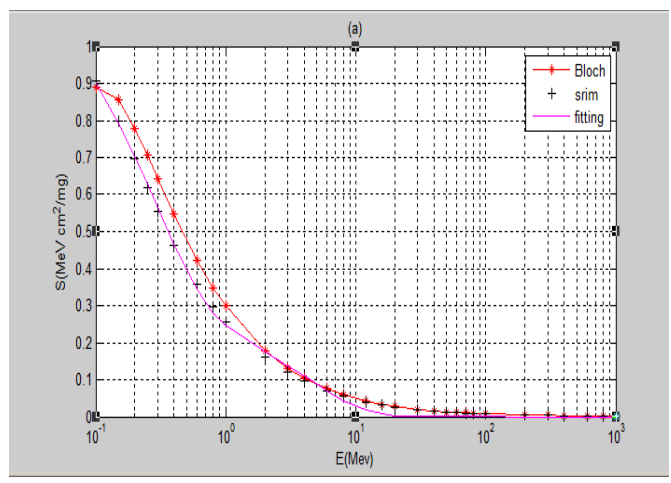

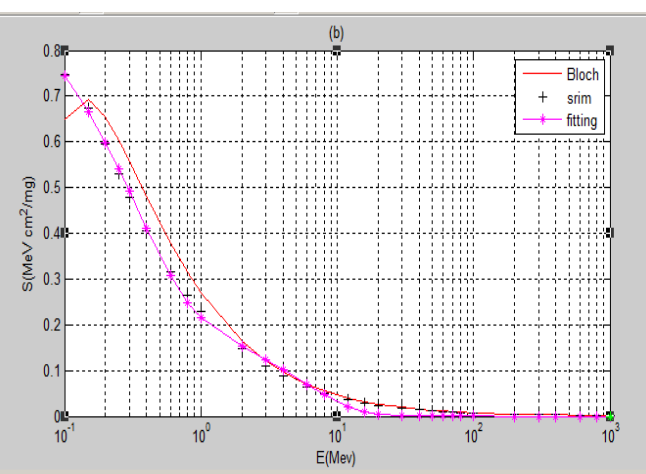

Figure 1: a. stopping power for protons in $\left(\mathrm{C}_{3} \mathrm{H}_{6}\right)$ b. stopping power for protons in $\left(\mathrm{C}_{22} \mathrm{H}_{10} \mathrm{~N}_{2} \mathrm{O}_{5}\right)$

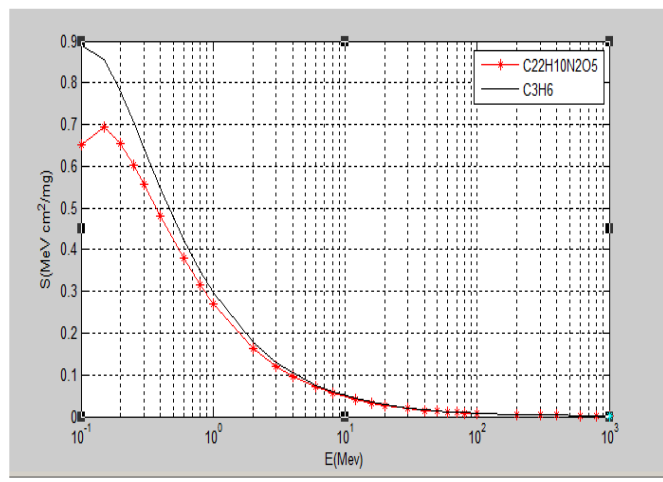

Figure 2: stopping power for protons in $\left(\mathrm{C}_{22} \mathrm{H}_{10} \mathrm{~N}_{2} \mathrm{O}_{5}\right)$ and $\left(C_{a} H_{6}\right)$

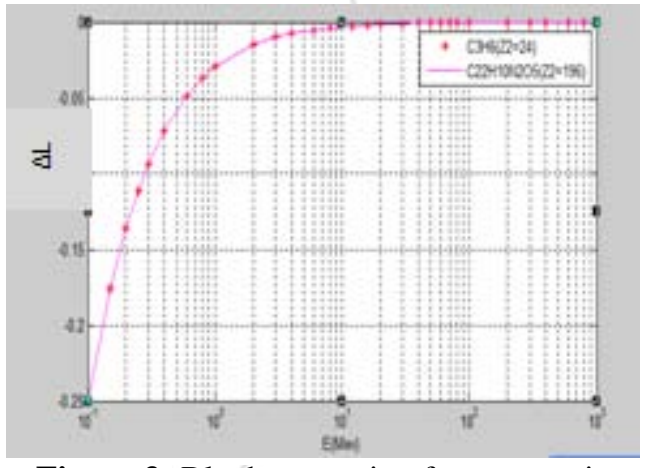

Figure 3: Bloch correction for protons in $C_{22} H_{10} N_{2} O_{5}\left(Z_{2}=280\right)$ and $C_{3} H_{6}\left(Z_{2}=24\right)$

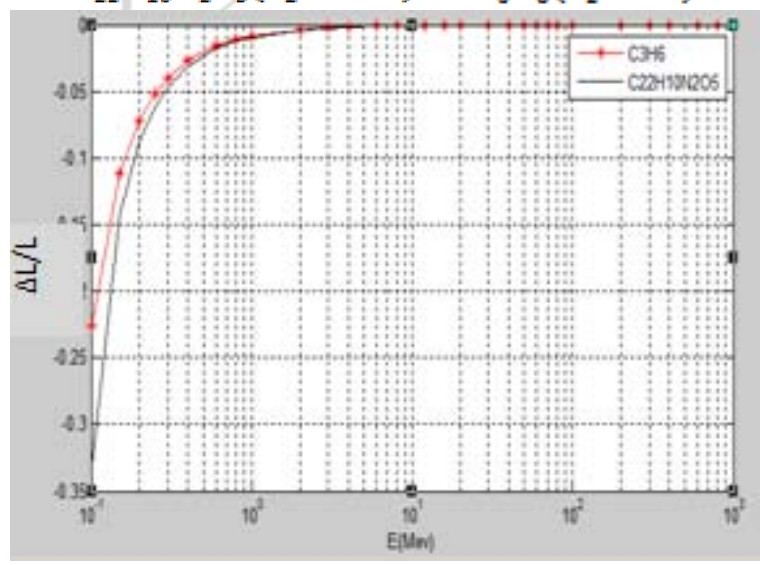

Figure 4: Percent contribution of Bloch correction to stopping number for protons in $\mathrm{C}_{3} \mathrm{H}_{6}\left(Z_{2}=24\right)$ and $\mathrm{C}_{22} \mathrm{H}_{10} \mathrm{~N}_{2} \mathrm{O}_{5}\left(\mathrm{Z}_{2}=280\right)$ 


\section{International Journal of Science and Research (IJSR) \\ ISSN (Online): 2319-7064}

Index Copernicus Value (2013): 6.14 | Impact Factor (2014): 5.611

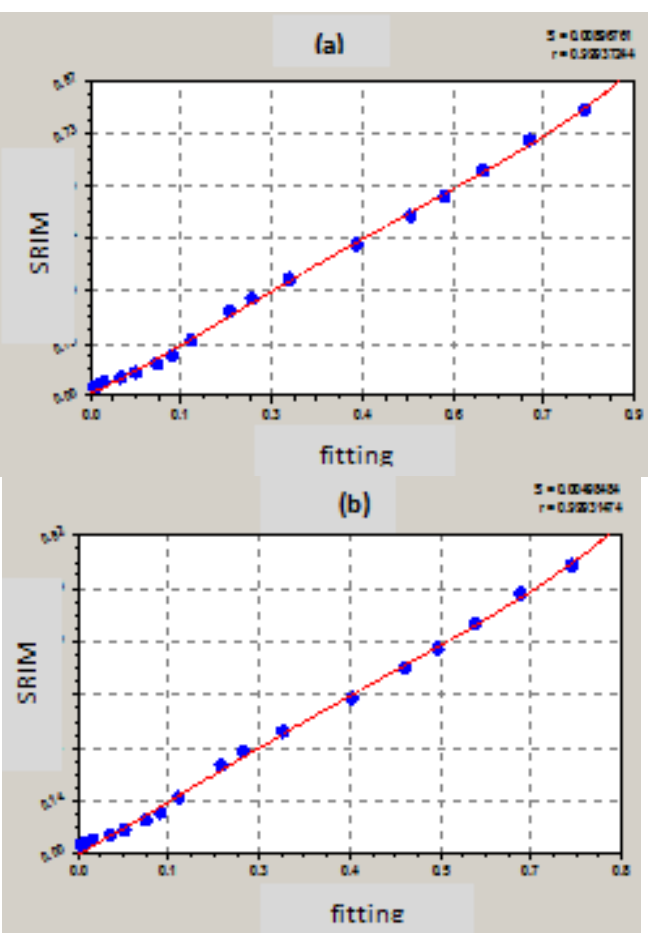

Figure 5: a. the Correlation Coefficient and the error margin between the semi experimental formula along with the results of SRIM 2012in $\left(C_{\mathrm{a}} H_{6}\right)$

b. the Correlation Coefficient and the error margin between the semi experimental formula along with the results of SRIM 2012in $\left(\mathrm{C}_{22} \mathrm{H}_{10} \mathrm{~N}_{2} \mathrm{O}_{5}\right)$
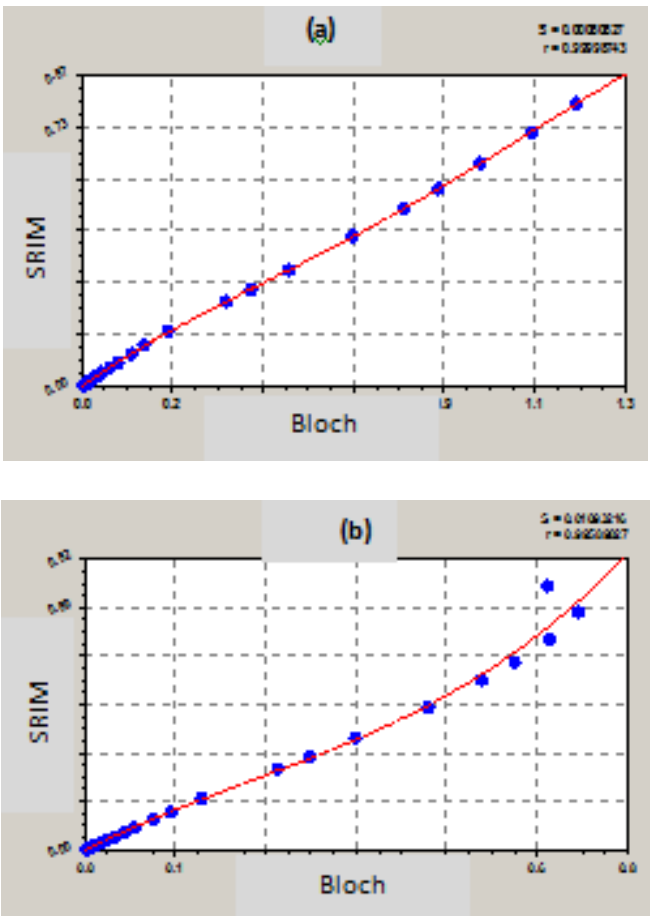

Figure 6: a. the Correlation Coefficient and the error margin between the results of Bloch formula with the results SRIM 2012 in $\left(\mathrm{C}_{3} \mathrm{H}_{6}\right)$

b. the Correlation Coefficient and the error margin between the results of Bloch formula with the results SRIM 2012 in $\left(\mathrm{C}_{22} \mathrm{H}_{10} \mathrm{~N}_{2} \mathrm{O}_{5}\right)$

\section{Conclusions}

The main stopping mechanism is the slowing down due to interactions with target atoms through excitations and ionizations of target electrons via Coulomb scattering. The Bethe theory of calculating stopping power of a point charge penetrating through matter at a non - relativistic elocity can be used over a wide energy interval for fast charged particles based on first Born approximation for atomic collision events which it provides astopping dependent on the square of projectile charge $\left(\mathrm{Z}_{1} \mathrm{e}\right)$.Deviations from the $\left(\mathrm{Z}_{1}^{2}\right)$ dependence are especially large at low velocity. For decreasing velocities, high order of $\left(\mathrm{Z}_{1}\right)$ terms $\left(\mathrm{Z}_{1}{ }^{3}\right.$ Barkas effect) and ( $Z_{1}^{4}$ Bloch correction) dependence in stopping power may be become important. Since the accuracy of this scheme deteriorates with increasing projectile charge and decreasing speed, it seemed reasonable to start at the opposite end, i. e. the classical limit (Bohr theory). Bohr pointed that the regime of validity of classical - orbit models and of quantal erturbation theory are roughly complementary. Bloch evaluated the differences between the classical (Bohr) and quantum-mechanical (Bethe) approaches for particles with velocities much larger than the target electrons. He showed that Bohr's harmonic oscillator approach was valid also in the quantum mechanics of a bound electron if the energy transferred was assumed to be the mean energy loss.For light ions (at high velocity and small Bohr's parameter $\mathrm{K}$ ), the Bloch correction becomes small and usually approaches to zero and the Bloch stopping number( $\left.\mathrm{L}_{\text {Bloch }}\right)$ approaches that of Bethe results eq.(4) but at low velocity, the Bethe theory becomes unphysical because of the negative stopping number, therefore the Bloch correction must be added to Bethe stopping number and arrives at the Bohr stopping number eq.(5) and provides the transition to the classical stopping power. Bloch correction depends upon the projectile and its velocity (energy) and are independent of the sign of the projectile charge $\left(\mathrm{Z}_{1} \mathrm{e}\right)$.

\section{References}

[1] Grande.P. L. and Schiwietz.G, Nucl.Inst.and Meth.In physics Research, B, 195 (2002) 55.

[2] Betz.H.D, Nucl.Inst.andMath.132 (1976) .

[3] Bohr. N, philos. Mag. 25 (1913) 10.

[4] Bethe .H, Ann. Physic.5 (1930) 325.

[5] Bloch.F, Ann.phys. 16 (1933) 285.

[6] Mott. N. F., Proc. R. Soc. London A, 124 (1929) 1856

[7] Lindhard .J and Sorensen .A. H, phys. Rev., A.53 (1996) 2443.

[8] Bichsel.H, Groom. D. E, and Klein. S.R, Phys. Rev., D. 66 (2002) 195.

[9] Weaver .B. A. and Westphal. A. J, Nucl. Inst. and Meth. In physics Research, B. 187 (2002) 285.

[10] P. Sigmund, .151 of Springer Series In Solid - State Sciences, .151 (2006).

[11]Bohr .N, Mat. Fys. Medd. Dan. Vid. Selsk. 18 (1948) 1.

[12] Sorensen .A. H, Phys. Rev., A . 55 (1997) 2896.

[13] Hans Bichsel, phys. Rev., A, Vol.41, N.7, pp.3642(1990).

[14]Ziegler. J.F and Ziegler .M.D, J.P. Biersack, Nucl.Instr.and Meth.in Phys.Res.B.268 (2010) 1818.

[15] Chaubey.A.K.and Gupta. H.V, Revuede Physique Appliquee To me 12 (1977) 321.

\section{Volume 5 Issue 3, March 2016}




\section{International Journal of Science and Research (IJSR) \\ ISSN (Online): 2319-7064}

Index Copernicus Value (2013): 6.14 | Impact Factor (2014): 5.611

[16] Northcliffe .L .C and Schilling, R. F, Nuclear Data A 7(1970)233.

[17] Whaling, W., Hand.Phys.34(1958) 193.

[18] Anderson, H.H., Hanke, C.C., Sorensen, H. and Vajda, P., Phys.Rev.153 (1967)338.

[19] Pierce, T.E.and Blann, M., Phys. Rev.173(1968)390.

[20] Booth, W.and Grant, I.S., Nucl. phys. 63(1965)481.

[21]H.H.Andersen and J.F.Ziegler, pergamon press, New York .2 (1977) .

[22] M.S.Abosaleh and A.M.Awed, Introduction to Statistics (2008). 